Article

\title{
A Robust GNSS/PDR Integration Scheme with GRU-Based Zero-Velocity Detection for Mass-Pedestrians
}

\author{
Dongpeng Xie ${ }^{1}$, Jinguang Jiang ${ }^{2, *}{ }^{\circledR}$, Jiaji Wu ${ }^{2}$, Peihui Yan ${ }^{2}{ }^{-}$, Yanan Tang ${ }^{2}$, Chao Zhang ${ }^{2}$ and Jingnan Liu ${ }^{2}$ \\ 1 Electronic Information School, Wuhan University, No. 129 Luoyu Road, Wuhan 430079, China; \\ dongpengxie@whu.edu.cn \\ 2 GNSS Research Center, Wuhan University, No. 129 Luoyu Road, Wuhan 430079, China; \\ aang_wu@whu.edu.cn (J.W.); phuiyan@whu.edu.cn (P.Y.); lucytang@whu.edu.cn (Y.T.); \\ chaozz@whu.edu.cn (C.Z.); jnliu@whu.edu.cn (J.L.) \\ * Correspondence: jinguang@whu.edu.cn
}

check for updates

Citation: Xie, D.; Jiang, J.; Wu, J.; Yan, P.; Tang, Y.; Zhang, C.; Liu, J. A Robust GNSS/PDR Integration Scheme with GRU-Based ZeroVelocity Detection for Mass-Pedestrians. Remote Sens. 2022, 14, 300. https:// doi.org/10.3390/rs14020300

Academic Editor: Kamil Krasuski

Received: 5 December 2021

Accepted: 4 January 2022

Published: 10 January 2022

Publisher's Note: MDPI stays neutral with regard to jurisdictional claims in published maps and institutional affiliations.

Copyright: (C) 2022 by the authors. Licensee MDPI, Basel, Switzerland. This article is an open access article distributed under the terms and conditions of the Creative Commons Attribution (CC BY) license (https:// creativecommons.org/licenses/by/ $4.0 /)$.

\begin{abstract}
Aiming at the problem of high-precision positioning of mass-pedestrians with low-cost sensors, a robust single-antenna Global Navigation Satellite System (GNSS)/Pedestrian Dead Reckoning (PDR) integration scheme is proposed with Gate Recurrent Unit (GRU)-based zero-velocity detector. Based on the foot-mounted pedestrian navigation system, the error state extended Kalman filter (EKF) framework is used to fuse GNSS position, zero-velocity state, barometer elevation, and other information. The main algorithms include improved carrier phase smoothing pseudo-range GNSS single-point positioning, GRU-based zero-velocity detection, and adaptive fusion algorithm of GNSS and PDR. Finally, the scheme was tested. The root mean square error (RMSE) of the horizontal error in the open and complex environments is lower than $1 \mathrm{~m}$ and $1.5 \mathrm{~m}$ respectively. In the indoor elevation experiment where the elevation difference of upstairs and downstairs exceeds $25 \mathrm{~m}$, the elevation error is lower than $1 \mathrm{~m}$. This result can provide technical reference for the accurate and continuous acquisition of public pedestrian location information.
\end{abstract}

Keywords: pedestrian navigation; GRU-based zero-velocity detection; integration navigation

\section{Introduction}

The joint application of low-cost inertial sensors, miniature global navigation satellite system (GNSS) receivers, and barometers has been one of the research hotspots in the field of navigation in the past decade [1-3]. Accurate and continuous pedestrian location information is widely used in professional applications such as armed police duty, field walking, pipeline maintenance, etc. The satellite navigation system can provide users with good location services in an open outdoor environment [4,5]. However, the system cannot work well in some scenarios where GNSS signals are unavailable $[6,7]$. Other information sources such as wireless local area networks (WLAN) [8], ultra-wideband (UWB) [9], radio frequency identification (RFID) [10], etc., can directly provide location information. However, there is a problem of signal occlusion in indoor scenarios. Generally, WiFi positioning and UWB positioning require extensive installation of signal receiving devices during the actual operation. Whether fingerprint positioning or trilateral measurement, WiFi positioning cannot be separated from offline surveying and mapping, limiting its application mode. Cameras [11] and radar [12] can improve the robustness of the system, however, these sensors only work effectively when there are enough features in the environment, which limits their application.

Some solutions such as multi-source information fusion have been proposed around the demand for continuous and reliable pedestrian navigation. Zhu et al. proposed a novel pedestrian navigation system (PNS) integrating RTK/Pseudolite/LAHDE/IMU, which uses manmade landmarks deployed at the entrance of the corridor to determine whether pedestrians are in an indoor corridor. If pedestrians do not follow a route with landmarks, 
the error will accumulate quickly [13]. Xin et al. presented a joint positioning scheme combining Bluetooth and inertial navigation systems, which provides meter-level positioning services without additional facilities [14]. Cavallo et al. introduced a solution which uses global position system (GPS) and Bluetooth to assist pedestrian trajectory calculation. This solution can continuously provide reliable positioning results for pedestrians in indoor or outdoor environments [15]. In [16], Polak et al. extended the power level measurement by using multiple anchors and multiple radio channels and focuses on the employing of machine learning methods to improve localization accuracy in an indoor environment under different conditions. In [17], Sun et al. integrated ultra-wideband technology and PDR to solve the problem of accumulated errors in inertial navigation systems. Compared with traditional methods, the positioning accuracy has been effectively improved.

As pedestrian movement patterns are complex, providing high-precision positioning in a complex environment is difficult by using a single navigation technology. With the continuous development of micro electro mechanical system (MEMS) technology, many sensor modules can be highly integrated into a small, low-power module, which provides natural advantages for public pedestrian navigation [18]. Magnetometer-assisted pedestrian navigation has long been widely studied $[19,20]$. To make magnetometer information play an active role in the system, the soft and hard magnetic effects must be calibrated, and the geomagnetic interference must be modeled and compensated [21,22]. Although the pedestrian navigation system based on inertial measurement units (IMU) can continuously provide positioning information, the positioning accuracy will quickly accumulate over time [23]. Mining pedestrian motion constraint information is of great significance for improving the performance of low-cost sensors. Pedestrians have natural zero-velocity information constraints during walking. Therefore, a zero-velocity update (ZUPT) algorithm can be widely used to suppress error accumulation [24]. In the zerovelocity phase of a gait cycle, the ZUPT algorithm combined with the extended Kalman filter (EKF) can effectively improve the positioning accuracy $[25,26]$.

For this reason, various gait detection schemes have been proposed [27-33]. Skog et al. used the output of IMU to offer a zero-velocity detection method based on a general likelihood ratio test (GLRT) [27]. Experiments show that this method achieves good results at a slow walking speed. Wang et al. proposed an adaptive stance-phase detection method based on inertial sensor, which deals with the measurement fluctuations in swing and stance phases differently and performs well in the presence of measurement fluctuations [28]. Liu et al. adjusted the threshold of zero-velocity detection dynamically according to the output of the accelerometer [29]. In [30], Wang et al. designed an algorithm to adjust the length of the window without changing the threshold. These methods do not clearly explain the connection with the existing zero-velocity detection methods based on maximum likelihood detection. In recent years, many researchers have proposed some detection methods based on artificial intelligence (hidden Markov model (HMM) [31], support vector machine (SVM) [32], long short term memory (LSTM) [33]) without threshold of zero-velocity detection in the pedestrian navigation system. Compared with traditional zero-velocity detection methods, these methods do not need to set specific thresholds. Although these methods require a lot of data to train the model and have a high computational cost, they are more robust than the traditional methods.

Although the traditional zero-velocity detection method can detect the zero-velocity state of pedestrians, it is hard to set the threshold of zero-velocity detection appropriately. The GNSS/SINS integrated navigation system can provide reliable positioning results in outdoor open environment, but it is not available in an indoor environment as the GNSS signal is interrupted. In this article, a robust single-frequency GNSS/PDR pedestrian navigation system is proposed. The error state Kalman filter is used to fuse GNSS positioning information, zero-velocity state, barometer elevation, and other information. The zero-velocity detector based on GRU can accurately detect the motion state of pedestrians. The adaptive robustness algorithm and lever arm model are used to make the system more 
robust. Experiments show that the proposed algorithm can obtain reliable positioning results in complex environments.

The rest of this article is arranged as follows. The second section presents the GNSS/PDR integration scheme architecture. In the third section, the main algorithms of the scheme are introduced, including improved carrier phase smoothing pseudo-range GNSS single-point positioning algorithm, GRU-based zero-velocity gait detection, and adaptive fusion algorithm of GNSS and PDR. The fourth section introduces the experiment results and analysis. The conclusion of this paper is given in the fifth section.

\section{GNSS/PDR Integration Scheme Architecture}

A robust integration scheme for single-frequency single-antenna GNSS/PDR in complex environments is proposed. It is designed to provide reliable, continuous, and accurate positioning results for low-cost mass pedestrian positioning in complex environments. Algorithmically, this integration scheme uses the error state EKF as the algorithm framework, considering the gait characteristics of pedestrian walking, and fusing GNSS position, barometer height, and other information. From the point of view of program realization, the high efficiency of calculation, the scalability of code, and modular programming are evaluated. The code structure is clear, which is convenient for developers to maintain, update, and collaborate. The scheme is mainly composed of the following five parts:

- Single-frequency data quality analysis, preprocessing, etc.

- Improve carrier phase smoothing GNSS single point positioning.

- GRU-based zero-velocity detector.

- $\quad$ Adaptive GNSS/PDR fusion positioning.

- Error analysis and visualization of positioning results.

The data fusion strategy flowchart is shown in Figure 1. Because of the unpredictability of pedestrian motion patterns and low sensor accuracy, the foot-mounted pedestrian navigation system is used as the basis, and the error state Kalman filter is used as the data fusion framework to deeply explore the performance of MEMS sensors. Motion constraint information is used to improve the positioning capability of the system. Figure 1 shows the single-frequency GNSS/PDR positioning algorithm. It mainly includes four parts: (1) GNSS positioning algorithm, (2) PDR algorithm, (3) GNSS/PDR positioning algorithm, (4) GRU-based gait detection algorithm.

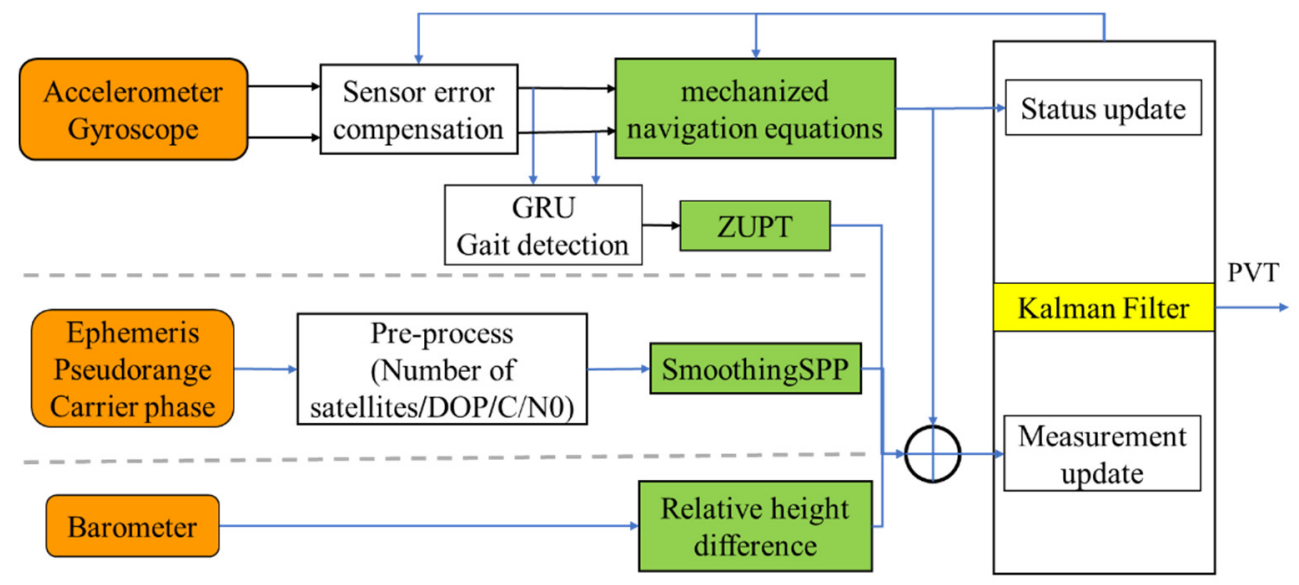

Figure 1. The data fusion strategy flowchart.

The overall architecture of the scheme is shown in Figure 2. The three sub-modules included are named INS module, GNSS module, and PNS module. INS module implements algorithms related to INS, GNSS module implements algorithms related to GNSS, and PNS module implements integrated navigation, self-adaptation, and result in display. Each sub-module contains its basic function and main algorithm. 


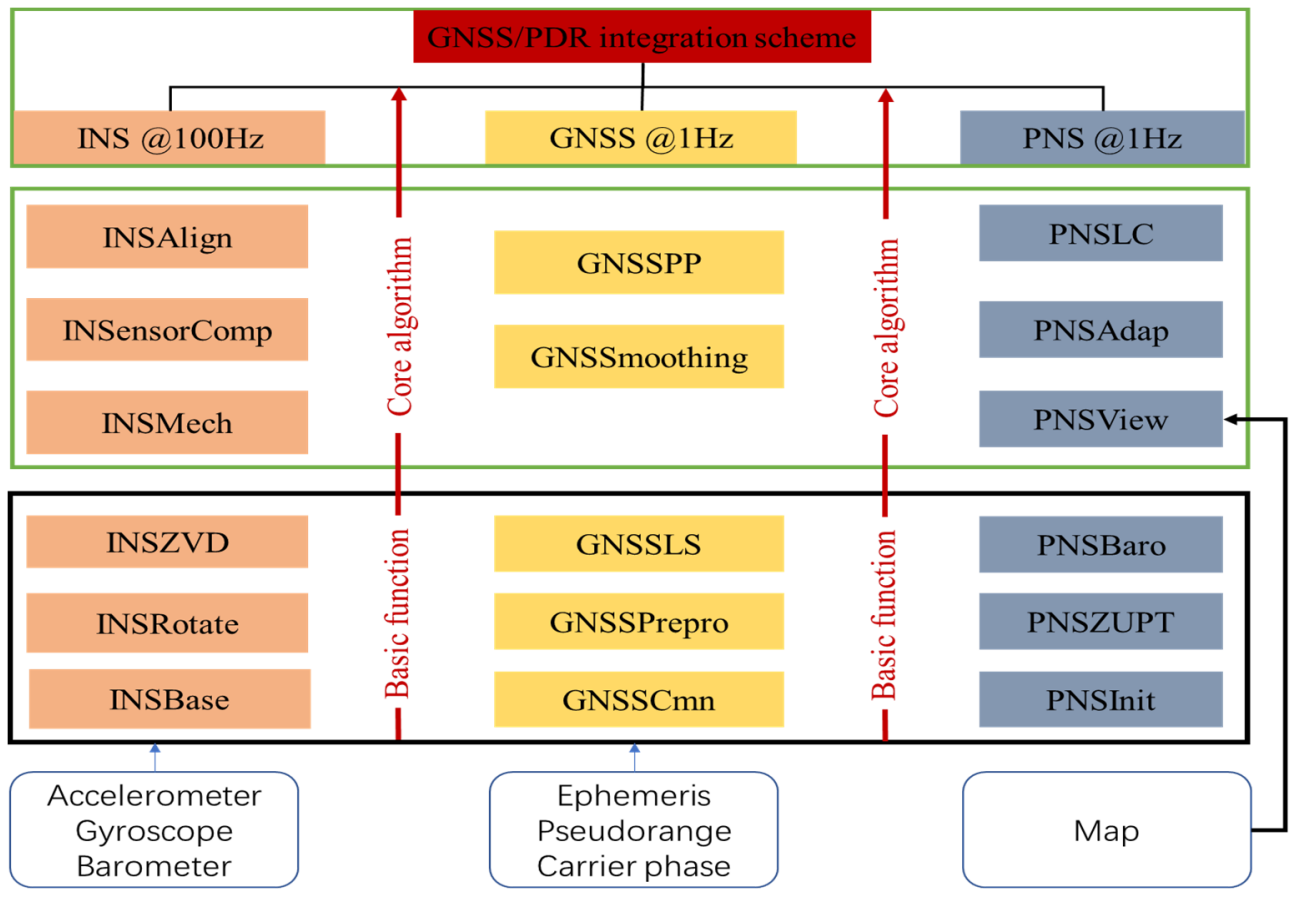

Figure 2. The overall architecture of the scheme.

\section{Fusion Method of GNSS and ZUPT-Aided PDR}

The algorithm flow chart is shown in Figure 1. This section shows the GNSS data preprocessing strategy, single-frequency single-antenna positioning algorithm, GRU-based zero-velocity detection, and GNSS/PDR integrated navigation algorithm.

\subsection{GNSS Data Preprocessing Strategy and Single-Frequency GNSS Positioning Algorithm}

Evaluating the data quality of observations from low-cost GNSS modules can provide a basis for the preprocessing of observations. The satellite visibility, carrier-to-noise ratio $\left(\mathrm{C} / \mathrm{N}_{0}\right)$, and pseudo-range noise are quantitatively evaluated and analyzed based on measured data. The number of visible satellites will directly affect the number of redundant observations in the data preprocessing. The $\mathrm{C} / \mathrm{N}_{0}$ is an essential indicator of the quality of the received observations. The GNSS/PDR integration scheme uses a variety of strategies to preprocess GNSS data, including detecting cycle slips and removing it, and for the problem that observations are prone to gross errors when the observations are at a low signal-to-noise ratio. Eliminate satellites below the threshold to avoid introducing abnormal observation information.

Hatch filtering is a standard processing method of traditional single-frequency carrier smoothing pseudo-range [34]. The traditional single-frequency hatch filter algorithm is prone to the problems of divergence of smoothing results and decreased accuracy due to the influence of ionospheric delay changes. In [35], Chebir et al. proposed a method based on applying specific transformations to the GNSS signals received in unfavorable environment, which can effectively receive and process GNSS signals in unfavorable environment. In [36], Park used a moving window algorithm to improve the original single-frequency smoothing pseudo-range algorithm. There are two forms of single-frequency smoothing, and the corresponding smoothing between epochs can be defined as:

$$
\begin{gathered}
\widehat{\rho}\left(t_{k}\right)=\frac{1}{k} \rho\left(t_{k}\right)+\left(1-\frac{1}{k}\right)\left[\widehat{\rho}\left(t_{k-1}\right)+\delta \phi\left(t_{k}, t_{k-1}\right)\right], k \geq 1 \\
\widehat{\rho}\left(t_{k}\right)=\left\{\begin{array}{l}
\frac{1}{k} \rho\left(t_{k}\right)+\left(1-\frac{1}{k}\right)\left[\widehat{\rho}\left(t_{k-1}\right)+\delta \phi\left(t_{k}, t_{k-1}\right)\right], 1 \leq k \leq M \\
\frac{1}{M} \rho\left(t_{k}\right)+\left(1-\frac{1}{M}\right)\left[\widehat{\rho}\left(t_{k-1}\right)+\delta \phi\left(t_{k}, t_{k-1}\right)\right], k>M
\end{array}\right.
\end{gathered}
$$


where $\rho(t)$ and $\phi(t)$ are the distances of the observed pseudo-range and carrier phase, respectively, and $M$ is the length of the filter, which is determined according to the smoothing time $\mathrm{T}$ and the observation sampling $\tau . \delta \phi\left(t_{k}, t_{k-1}\right)$ is the difference operator.

\subsection{GRU-Based Zero-Velocity Detection}

Recurrent neural network (RNN) is now widely used in time series-based forecasting tasks (pedestrian trajectory prediction [37], vehicle trajectory prediction [38], etc.). GRU is a special RNN with the ability to learn long-term dependency.

The structure of the GRU is shown in Figure 3, which is composed of an update gate $\left(\boldsymbol{\Gamma}_{u}\right)$ and a reset gate $\left(\boldsymbol{\Gamma}_{r}\right)$. The larger the value of the update gate, the more the state information of the previous moment is brought in. The reset gate controls how much information is conveyed to the current candidate set $\left(\widetilde{c}^{<t>}\right)$. The smaller the reset gate is, the less information is passed to the previous state. The detailed update formula is as follows:

$$
\begin{gathered}
\Gamma_{r}=\sigma\left(W_{r}\left[c^{<t-1>}, x^{<t>}\right]+b_{r}\right) \\
\Gamma_{u}=\sigma\left(W_{u}\left[c^{<t-1>}, x^{<t>}\right]+b_{u}\right) \\
\widetilde{c}^{<t>}=\tanh \left(W_{c}\left[\Gamma_{r} \odot c^{<t-1>}, x^{<t>}\right]+b_{c}\right) \\
c^{<t>}=\Gamma_{u} \odot \widetilde{c}^{<t>}+\left(\mathbf{I}-\Gamma_{u}\right) \odot c^{<t-1>}
\end{gathered}
$$

where $\odot$ represents the relative position of the objects before and after multiplication. $\sigma$ represents the sigmoid activation of the following objects. $c^{<t-1>}$ represents the activation value of the previous time step. $\boldsymbol{W}_{c}, \boldsymbol{W}_{u}, \boldsymbol{W}_{\boldsymbol{r}}$ correspond to the weight matrix of each gate respectively. $\boldsymbol{b}_{\boldsymbol{r}}, \boldsymbol{b}_{\boldsymbol{z}}, \boldsymbol{b}_{\boldsymbol{h}}$ are the bias vectors of reset gate, update gate, and hidden unit respectively.

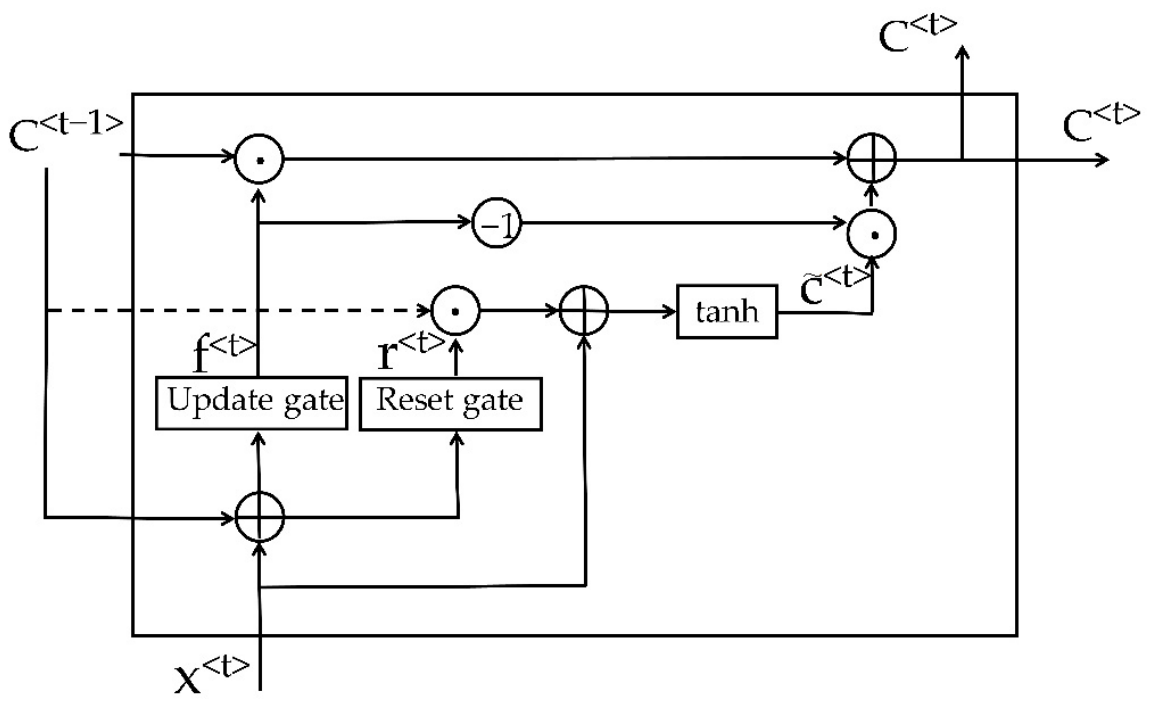

Figure 3. GRU block architecture.

GRU-based zero-velocity detectors need to collect IMU data from different objects to train the model. Currently, there is no standard method to generate labels. Some existing techniques (manual, pressure sensor, high-precision sports state capture system) are proposed to produce reliable zero-velocity information. In this paper, the results of RTK/INS combination are used as reference trajectories, adjust the threshold of the GLRT detector to produce the smallest RMSE, and use the zero-velocity state by the GLRT detector with an optimal threshold as a label.

The structure of the zero-velocity detector is shown in Figure 4, which is mainly composed of three GRU layers, two drop layers, and one fully connected layer (FC). Each 
layer of GRU has 100 neurons. To avoid overfitting, the drop layer is set to 0.2. The FC layer uses a sigmoid function as the activation function to map the model output to within $(0,1)$. It is assumed that current state is zero-velocity when the model output is greater than 0.8 , otherwise, current state is non-zero-velocity. During the training process, 50 consecutive IMU data (specific force, angular velocity) constitute one sample, and a data set consisting of 300,000 samples is used to train this model. Each sample has an individual label which represents the zero-velocity state corresponding to the last time step. The Adam optimizer [39] is used to optimize the model with 100 iterations. The loss function of the model is defined as follows:

$$
\text { Loss }=-\frac{1}{N} \sum_{i}^{N} y_{i} \log \left(p_{i}\right)+\left(1-y_{i}\right) \log \left(1-p_{i}\right)
$$

where $N$ represents the total number of training samples and $p_{i}$ represents the label output of the $i$-th sample.

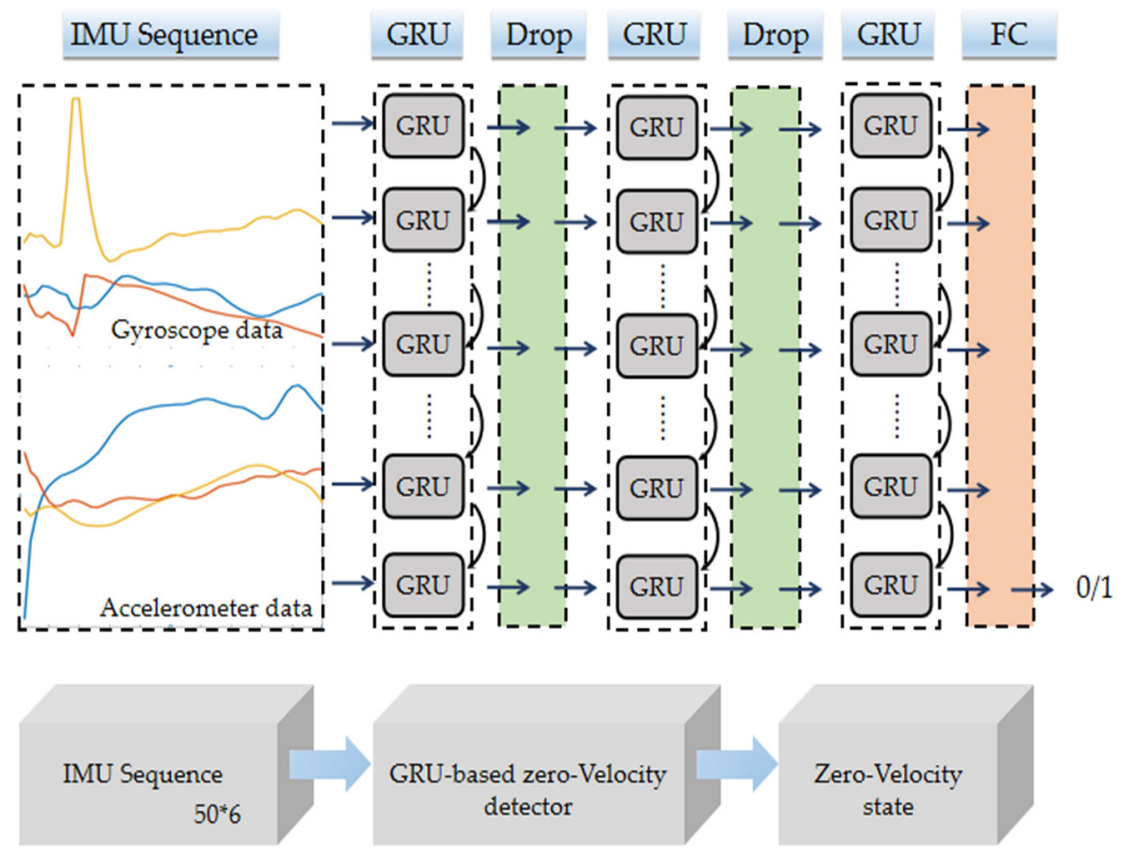

Figure 4. The structure of zero-velocity detection based on GRU.

\subsection{Robust EKF Design for GNSS/PDR Integration}

The error state EKF is used as the data fusion framework. The 15-dimensional error state vector is summarized as follows:

$$
\delta \boldsymbol{x}=\left[\begin{array}{llll}
\delta \boldsymbol{p} & \delta \boldsymbol{v} & \delta \boldsymbol{\varphi} & \delta \boldsymbol{b}_{\mathrm{g}} \delta \boldsymbol{b}_{\boldsymbol{a}}
\end{array}\right]
$$

where $\delta \boldsymbol{p}, \delta \boldsymbol{v}, \delta \boldsymbol{\varphi}, \delta \boldsymbol{b}_{\boldsymbol{g}}, \delta \boldsymbol{b}_{\boldsymbol{a}}$ are all three-dimensional vectors and denote position, velocity, attitude, gyroscope bias, and accelerometer bias. The discrete-time error model of INS can be defined in matrix form as:

$$
\delta \boldsymbol{x}_{k, k-1}=\boldsymbol{F}_{k, k-1} \delta \boldsymbol{x}_{k}+\boldsymbol{G}_{k, k-1} \boldsymbol{W}_{k-1}
$$

where $\boldsymbol{F}_{k, k-1}$ is the state transition matrix, $\boldsymbol{G}_{k, k-1}$ is process noise-driven matrix, and $\boldsymbol{W}_{k-1}$ is process noise, which is assumed to be Gaussian white noise with zero mean; the state transition and noise gain matrices can be written as: 


$$
\begin{gathered}
\boldsymbol{F}=\left[\begin{array}{ccccc}
\boldsymbol{I}_{3} & \mathbf{0}_{3} & \mathbf{0}_{3} & \mathbf{0}_{3} & -C_{b}^{n} \Delta t \\
\Delta t\left(\boldsymbol{C}_{b}^{n} f^{b}\right) \times & \boldsymbol{I}_{3} & \mathbf{0}_{3} & \boldsymbol{C}_{b}^{n} \Delta t & \mathbf{0}_{3} \\
\mathbf{0}_{3} & \Delta t \boldsymbol{I}_{3} & \boldsymbol{I}_{3} & \mathbf{0}_{3} & \mathbf{0}_{3} \\
\mathbf{0}_{3} & \mathbf{0}_{3} & \mathbf{0}_{3} & \boldsymbol{B}_{g} & \mathbf{0}_{3} \\
\mathbf{0}_{3} & \mathbf{0}_{3} & \mathbf{0}_{3} & \mathbf{0}_{3} & \boldsymbol{B}_{a}
\end{array}\right] \\
\boldsymbol{b}_{g}=\boldsymbol{I}_{3}+\Delta t \cdot \operatorname{diag}\left(-\frac{1}{\tau_{g}}\right) \\
\boldsymbol{b}_{a}=\boldsymbol{I}_{3}+\Delta t \cdot \operatorname{diag}\left(-\frac{1}{\tau_{a}}\right) \\
\quad\left[\begin{array}{cccc}
\mathbf{0}_{3} & \mathbf{0}_{3} & \mathbf{0}_{3} & \mathbf{0}_{3} \\
\boldsymbol{C}_{b}^{n} \Delta t & \mathbf{0}_{3} & \mathbf{0}_{3} & \mathbf{0}_{3} \\
\mathbf{0}_{3} & \boldsymbol{C}_{b}^{n} \Delta t & \mathbf{0}_{3} & \mathbf{0}_{3} \\
\mathbf{0}_{3} & \mathbf{0}_{3} & \boldsymbol{I}_{3} \Delta t & \mathbf{0}_{3} \\
\mathbf{0}_{3} & \mathbf{0}_{3} & \mathbf{0}_{3} & \boldsymbol{I}_{3} \Delta t
\end{array}\right]
\end{gathered}
$$

where $(\cdot) \times$ represents the skew-symmetric matrix of a vector. $I_{p}$ and $0_{p}$ denote $p \times p$ identity matrix and zero matrices respectively. The bias errors of gyros and accelerometers can be expressed as first-order Gauss-Markov processes with the correlation time $\tau_{g}, \tau_{a}$.

When the observations (including the GNSS positioning results, the relative height difference of the barometer, and the zero-velocity information) are available, the measurement equation is constructed:

$$
\delta z_{k}=\boldsymbol{H}_{k} \delta \widehat{\boldsymbol{x}}_{k, k-1}
$$

GNSS positioning is the main factor that determines the absolute positioning accuracy of the system and suppresses the error of the inertial sensor. At the same time, the lever arm error cannot be ignored, and the position observation equation is:

$$
\delta z_{r}=\widehat{r}_{i n s}^{n}-\widehat{r}_{g n s s}^{n}
$$

Use the barometer elevation change as the observation equation to update the elevation:

$$
\delta z_{h}=\left(\boldsymbol{H}_{\text {baro }}^{k}-\boldsymbol{H}_{\text {baro }}^{k_{0}}\right)-\left(\boldsymbol{H}_{\text {ins }}^{k}-\boldsymbol{H}_{\text {ins }}^{k_{0}}\right)
$$

where $\boldsymbol{H}_{\text {baro }}^{k}$ and $\boldsymbol{H}_{\text {baro }}^{k_{0}}$ is the barometer elevation of the current epoch and the previous epoch, respectively. $\boldsymbol{H}_{i n s}^{k}$ and $\boldsymbol{H}_{i n s}^{k_{0}}$ is the elevation of the INS recursive of the current epoch and the previous epoch, respectively.

ZUPT is an effective means to control the accumulation of velocity errors. When the GRU recurrent neural network is used to detect that it is in a static state, the zero-velocity observation model is constructed:

$$
\delta \boldsymbol{z}_{v}=\widehat{\boldsymbol{v}}_{i n s}^{n}-\left[\begin{array}{lll}
0 & 0 & 0
\end{array}\right]^{T}
$$

\subsection{Lever Correction}

When carrying out the walking experiment, the IMU is fixed on the surface of the shoe, and the GNSS receiver antenna is set on the top of the head. The lever arm is constantly changing and cannot be ignored. It is not recommended to put the lever arm in the state vector as a parameter to be estimated, which not only increases the complexity of the calculation but also there is no way to evaluate whether the estimated value of the lever arm is correct. In the zero-velocity interval, the distance between the GNSS antenna and the IMU measurement center in the horizontal direction is much lower than the position error of GNSS single-point positioning result. The lever length in the vertical direction is 
equal to the height of the human subject. In the non-zero-velocity interval, it is modeled as a pendulum, as shown in Figure 5. A lever arm is expressed as:

$$
\text { lever } \operatorname{arm}=\left[\begin{array}{lll}
l_{2} \times \sin \theta & 0 & l_{1}+l_{2} \times \cos \theta
\end{array}\right]
$$

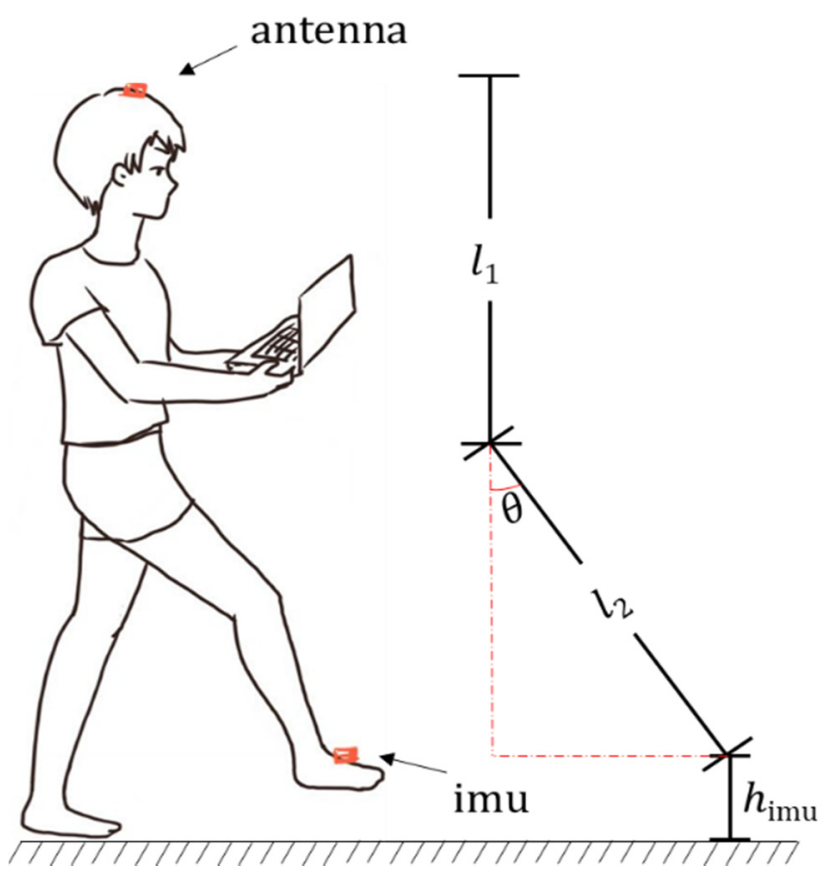

Figure 5. Lever arm model.

In the above Figure $5, l_{1}$ represents the height from the top of the head to the waist, $l_{2}$ is the length of the legs, which can be accurately known, and $\theta$ is an unknown parameter, which can be obtained by solving the essential trigonometric function.

The error caused by the inaccurate lever arm modeling can be equivalent to the GNSS single-point positioning error, which can be effectively compensated by an adaptive filter.

\subsection{Adaptive Kalman Filter}

The GNSS outliers appear in the harsh environment due to the poor GNSS observation quality. However, the standard Kalman filter cannot solve this problem, which increases the positioning error of the GNSS/INS integrated system. Innovation-based adaptive estimation (IAE) filter is a popular filter to reduce the influence of outliers, which is adopted in this paper.

By calculating the average value of the covariance of the innovation vector of the previous $\mathrm{N}$ epochs, the estimate of the covariance of the innovation vector at current epoch and the estimation of the observation vector covariance matrix can be obtained:

$$
\begin{aligned}
\widehat{\Sigma}_{\overline{\boldsymbol{V}}_{k}} & =\frac{1}{N} \Sigma_{j=0}^{N} \overline{\boldsymbol{V}}_{k-j} \overline{\boldsymbol{V}}_{k-j}^{T} \\
\widehat{\Sigma}_{k} & =\widehat{\Sigma}_{\overline{\boldsymbol{V}}_{k}}-\boldsymbol{F}_{k} \Sigma_{\overline{\boldsymbol{X}}_{k}} \boldsymbol{F}_{k}^{T}
\end{aligned}
$$

where $\widehat{\Sigma}_{\bar{V}_{k}}$ denotes the covariance matrix of the state one-step prediction vector, and $V_{k}$ denotes the innovation vector. This calculates the ratio of the trace of $\widehat{\Sigma}_{k}$ to the trace of preset observation vector covariance matrix $\Sigma_{k}$. When the position result of GNSS is an outlier, this ratio will be greater than 1 . The greater one between this ratio and one is taken as the adaptive factor: 
The adaptive factor is used to expand the covariance matrix of the observation vector at the current epoch, which reduces the influence of GNSS outliers and increases the position accuracy of the GNSS/INS integrated system.

The paper studies the single-frequency single-system single-antenna pseudo-range single-point positioning assisted foot-mounted micro-inertial pedestrian navigation method. When carrying out the walking experiment, the MTi-G-710 is fixed on the shoe surface, the GNSS antenna is fixed on the top of the head, and the collected data is used to test the accuracy of carrier phase smoothing pseudo-range SPP positioning and the effect of SPP/SINS combination.

\section{Results}

MTi-G-710 produced by the Dutch company Xsens is selected as the experimental device, which integrates a three-axis gyroscope, a three-axis accelerometer, and a barometer. The performance parameters of the gyroscope and accelerometer are shown in Table 1. The experimental GNSS module is the mosaic-X5 produced by Septentrio, Belgium, which is used to track all visible supporting satellites at the same time. The sampling rate of the IMU was set to 100. The data of the experiment is available for download here: https:/ / github.com/laotouyu123/data_set.git, accessed on 6 November 2021.

Table 1. Specifications of inertial sensors.

\begin{tabular}{ccc}
\hline Parameters & Gyroscope & Accelerometer \\
\hline Full Scale & $625^{\circ} / \mathrm{s}$ & $18 \mathrm{~g}$ \\
Bias stability & $10^{\circ} / \mathrm{h}$ & $40 \mathrm{ug}$ \\
Noise density & $0.01^{\circ} / \mathrm{s} / \sqrt{\mathrm{Hz}}$ & $80 \mu \mathrm{g} / \sqrt{\mathrm{Hz}}$ \\
g-sensitivity & $0.003^{\circ} / \mathrm{s} / \mathrm{g}$ & - \\
Non-orthogonal error & $0.05^{\circ}$ & $0.05^{\circ}$ \\
Non-linearity & $0.01 \%$ & $0.01 \%$ \\
\hline
\end{tabular}

All parameters in the table are typical values at $25^{\circ} \mathrm{C}$.

\subsection{GRU-Based Zero-Velocity Detection Algorithm Performance Verification}

In order to verify the performance and generalization ability of the GRU-based zerovelocity detection method, ten experimenters carried out walking experiments on the same path at three walking speeds: slow, normal, and fast. The zero-velocity detection result of one of the experimenters is shown in Figure 6.

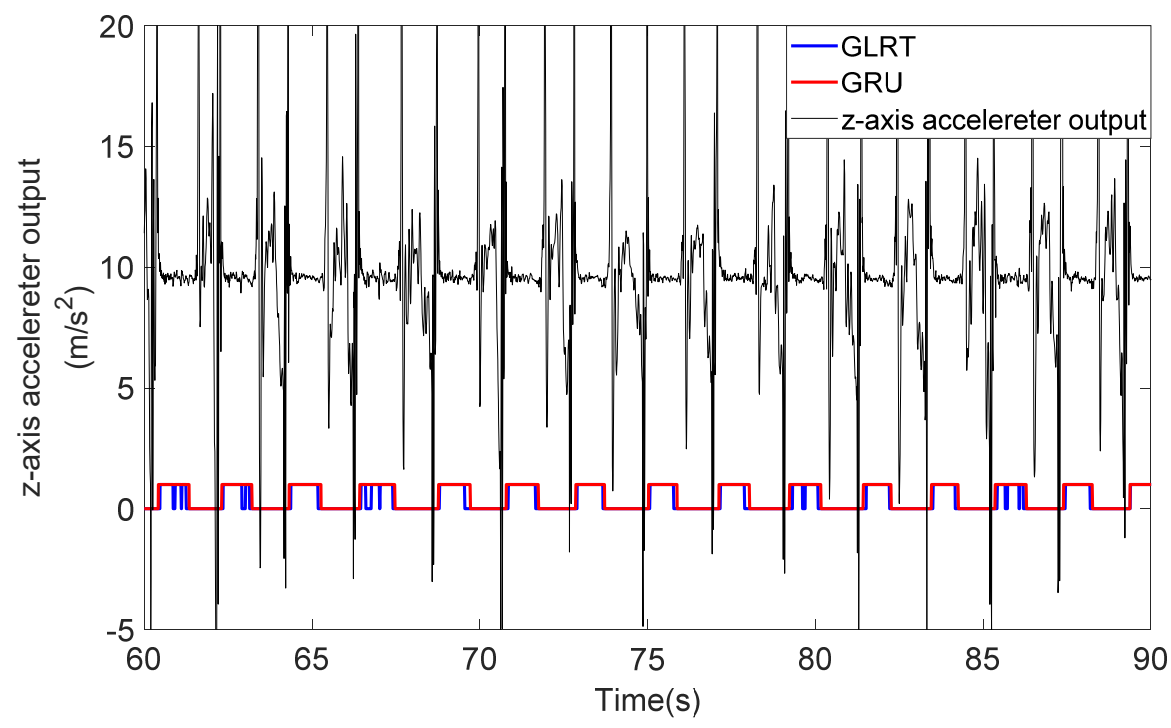

Figure 6. Zero-velocity detection result. 
As shown in Figure 6, the GLRT method can effectively detect the zero-velocity most of the time. However, some incorrect zero-velocity results are detected by GLRT when the feet of the experimenter are moving, which will decrease the positioning precision of the PDR algorithm. The GRU method can detect the zero-velocity correctly all the time as the red curve shows in Figure 6, which guarantees the availability of zero-velocity information.

In order to verify the performance of the GRU-based zero-velocity detection method, a close-loop experiment is carried out, in which an experimenter walked 10 times along a rectangular trajectory. The trajectories obtained by two zero-velocity detect methods are shown in Figure 7. It can be seen from Figure 7 that the GLRT method obtained larger position error in the end point because of the incorrect zero-velocity information.

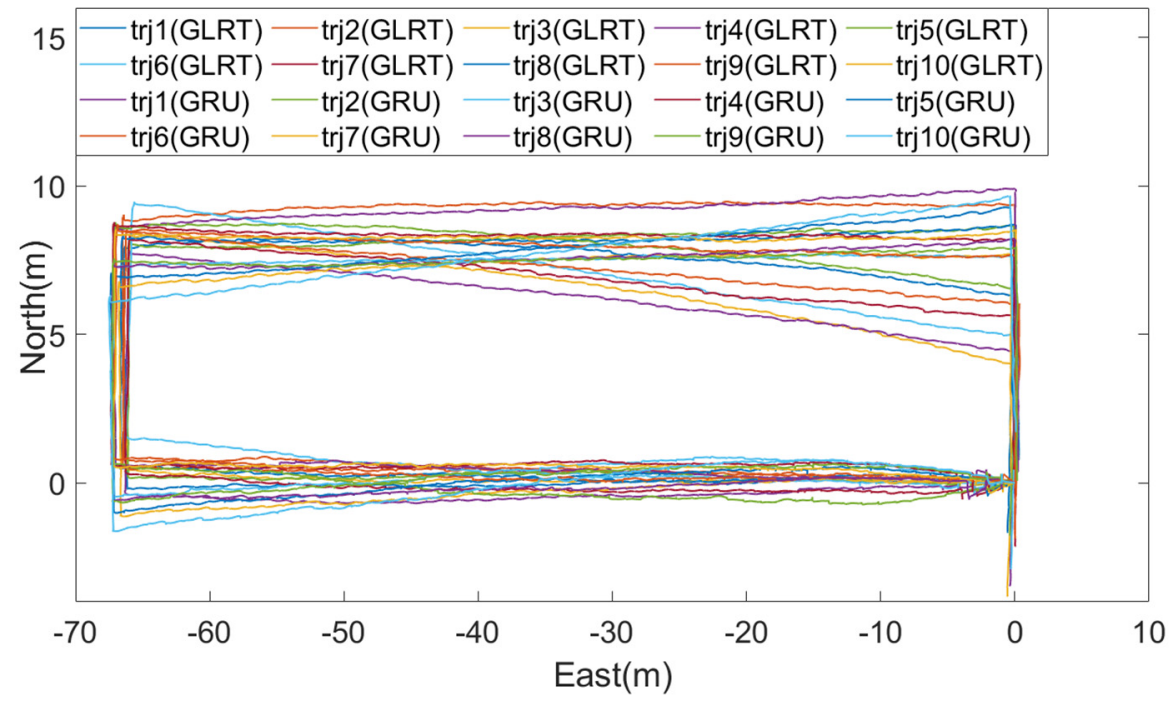

Figure 7. Trajectories obtained by different zero-velocity detection methods.

The closed-loop errors of all trajectories obtained by the GLRT method and the GRU method are listed in Table 2. The minimum average closed-loop position error of the traditional GLRT zero-velocity detection method is $1.42 \mathrm{~m}$, while the average closed-loop position error of the proposed method is $0.89 \mathrm{~m}$, which means the proposed method performs better than the traditional algorithm.

Table 2. Closed loop position error (m) obtained by different zero-velocity detection methods.

\begin{tabular}{ccccccc}
\hline \multirow{2}{*}{ Index } & \multicolumn{5}{c}{ GLRT } & \multirow{2}{*}{ GRU } \\
\cline { 2 - 6 } & $\gamma(10,000)$ & $\gamma(15,000)$ & $\gamma(20,000)$ & $\gamma(25,000)$ & $\gamma(30,000)$ & \\
\hline Max & 3.98 & 3.76 & 0.59 & 3.73 & 3.82 & 1.77 \\
Min & 0.98 & 0.04 & 0.43 & 0.43 & 0.25 & 0.11 \\
Mean & 2.28 & 1.77 & 1.44 & 1.42 & 1.91 & 0.89 \\
\hline
\end{tabular}

\subsection{The Proposed Algorithm Performance Verification under Open Environment}

To verify the proposed algorithm performance under open environment, a data set (249 s in total) was collected under an open environment at Youyi Square, Faculty of Informatics, Wuhan University, Wuhan. The experimenter walks along the edge of Youyi Square during the experiment.

In the Kalman filter algorithm for pedestrian positioning, the relevant parameters are set as follows: initial speed error is $0.01 \mathrm{~m} / \mathrm{s}$, the initial position error is $0.01 \mathrm{~m}$, the initial value of acceleration offset is $0.03 \mathrm{~m} / \mathrm{s}^{2}$, the initial value of gyroscope offset is $0.3^{\circ} / \mathrm{s}$, accelerometer noise is $\delta_{\mathrm{a}}=0.5 \mathrm{~m} / \mathrm{s}^{2}$, gyro noise is $\delta_{\mathrm{g}}=1^{\circ} / \mathrm{s}$, accelerometer bias driving noise $\delta_{\mathrm{b}_{\mathrm{a}}}=10^{-4} \mathrm{~m} / \mathrm{s}^{2}$, gyroscope bias driving noise $\delta_{\mathrm{b}_{\mathrm{g}}}=10^{-5} \mathrm{rad} / \mathrm{s}$. 
Compare the positioning result of the following four schemes: Scheme 1, use the GLRT method to detect the zero-velocity interval; Scheme 2, use the GRU method to detect the zero-velocity interval; Scheme 3, the proposed algorithm; Scheme 4, RTK. The trajectories of these schemes are shown in Figure 8.

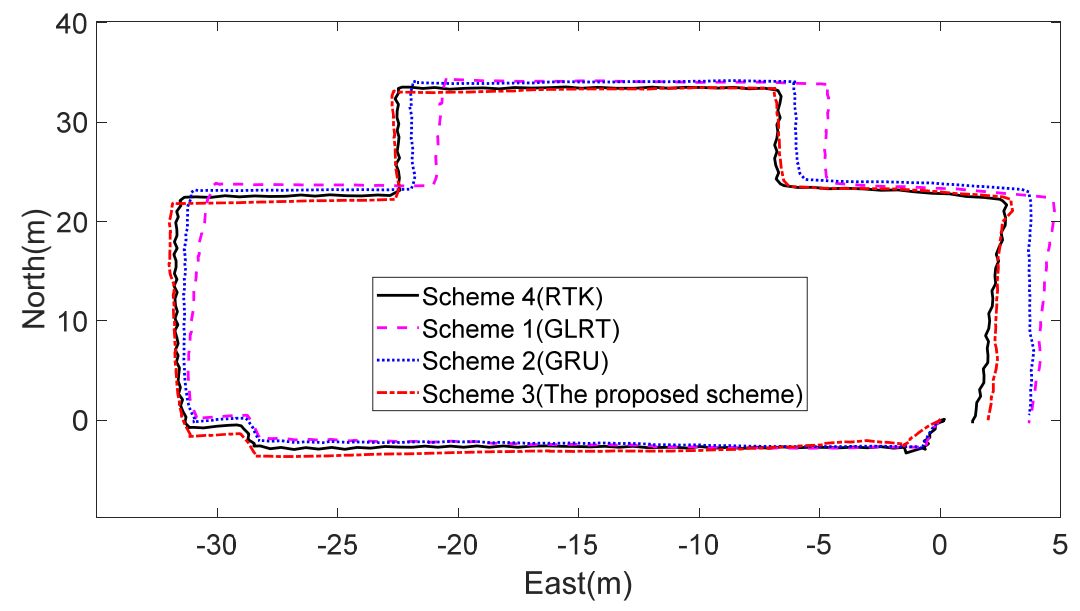

Figure 8. 2D positioning trajectories by different methods.

Under the open environment, RTK's positioning error is small, so the RTK position result of Scheme 4 can be used as a reference truth value. The statistical results of three schemes are shown in Table 3. It can be seen that the RMSE and maximum errors of Scheme 3 are more minor than those of the other two methods. The RMSE of these three schemes are $1.643 \mathrm{~m}, 1.042 \mathrm{~m}$, and $0.543 \mathrm{~m}$ respectively. The maximum errors of the three schemes are $2.631 \mathrm{~m}, 2.54 \mathrm{~m}$, and $1.03 \mathrm{~m}$ respectively. Compared with Schemes 1 and 2, RMSE of the proposed scheme are decreased by $67 \%$ and $48 \%$ respectively. In terms of maximum errors, compared with other two schemes, the maximum errors of the proposed scheme are decreased by $61 \%$ and $59 \%$ respectively. In order to more clearly reflect the performance of the proposed scheme, the Cumulative Distribution Function (CDF) of the horizontal error is shown in Figure 9. It can be seen from Figure 9 that $99 \%$ of the horizontal error of the proposed scheme is smaller than $1 \mathrm{~m}$, while that of the other two schemes is $33 \%$ and $63 \%$.

Table 3. Position error (m) obtained by different schemes.

\begin{tabular}{ccc}
\hline & Maximum Error & RMSE \\
\hline Scheme 1 & 2.63 & 1.63 \\
Scheme 2 & 2.54 & 1.04 \\
Scheme 3 & 1.03 & 0.54 \\
\hline
\end{tabular}

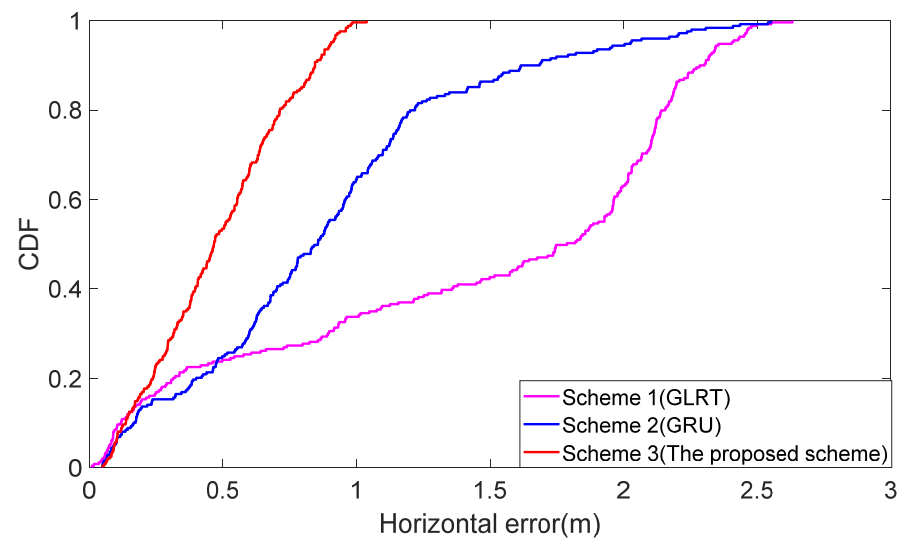

Figure 9. Cumulative distribution function by different methods. 


\subsection{The Proposed Algorithm Performance Verification under Complex Environment}

To verify the proposed algorithm performance under complex environment, a data set was collected in the playground of the Faculty of Information Science of Wuhan University, Wuhan. The experimenter walked along the outermost periphery of the playground. There are many interferences in the trajectory, including trees and houses. The experiment environment is shown in Figure 10.

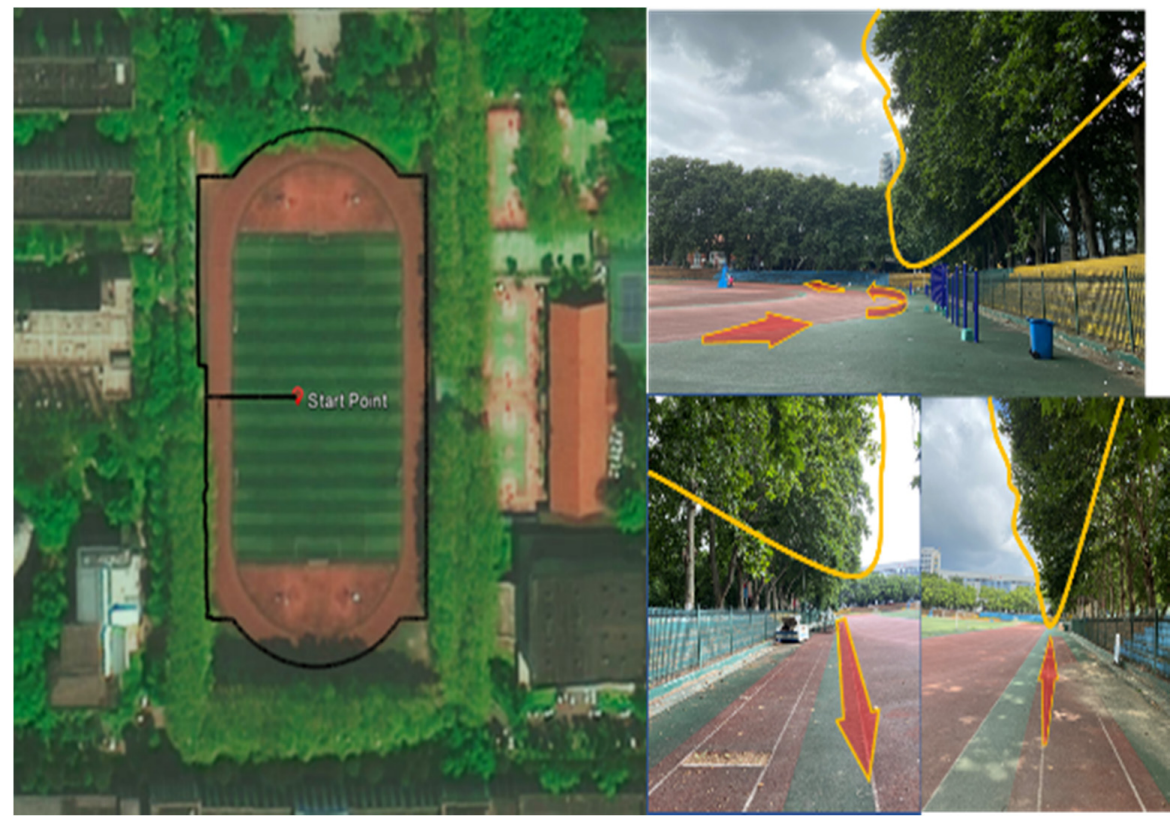

Figure 10. The experiment environment.

The $C / N_{0}$ of the satellite signal is shown in Figure 11. Due to the unsatisfactory observation environment, the number of satellites is maintained at about 8 . However, the loss of satellite signal occurred frequently; the carrier-to-noise ratio of a few satellites is lower than $30 \mathrm{~dB}-\mathrm{Hz}$ most of the time, such as G29 and G15. The position and position covariance results calculated by the carrier smoothing pseudo-range SPP are used in the measurement update of the EKF in the proposed scheme, and the adaptive algorithm is used for quality control.

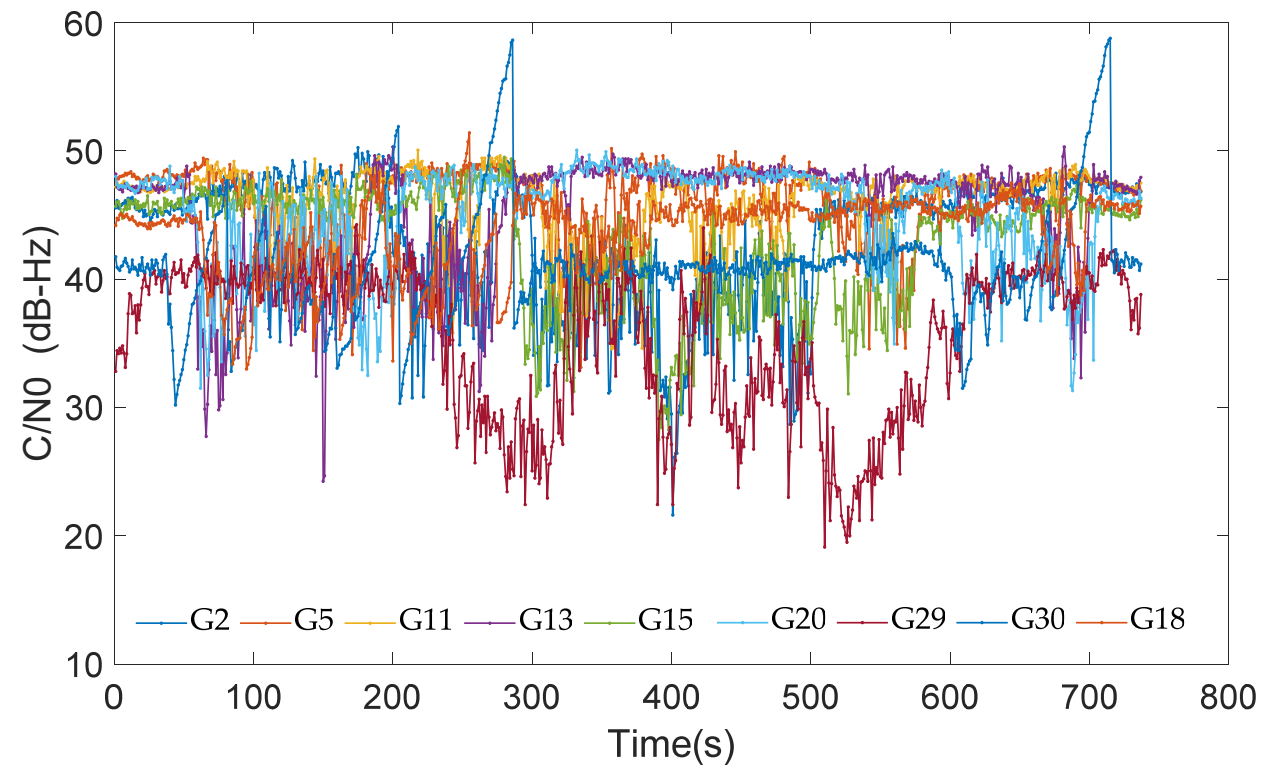

Figure 11. Carrier-to-noise ratio of satellite signal. 
Eighteen key positions of the trajectory are selected as reference points; their position coordinate is accurately determined. Five schemes are compared. Scheme 1: use GLRT method to detect the zero-velocity interval; Scheme 2: use GRU method to detect the zero-velocity interval; Scheme 3: GNSS/PDR without adaptive algorithm; Scheme 4: the proposed algorithm; Scheme 5: RTK solution provided by mosaic-X5. The trajectories of these five schemes are shown in Figure 12.

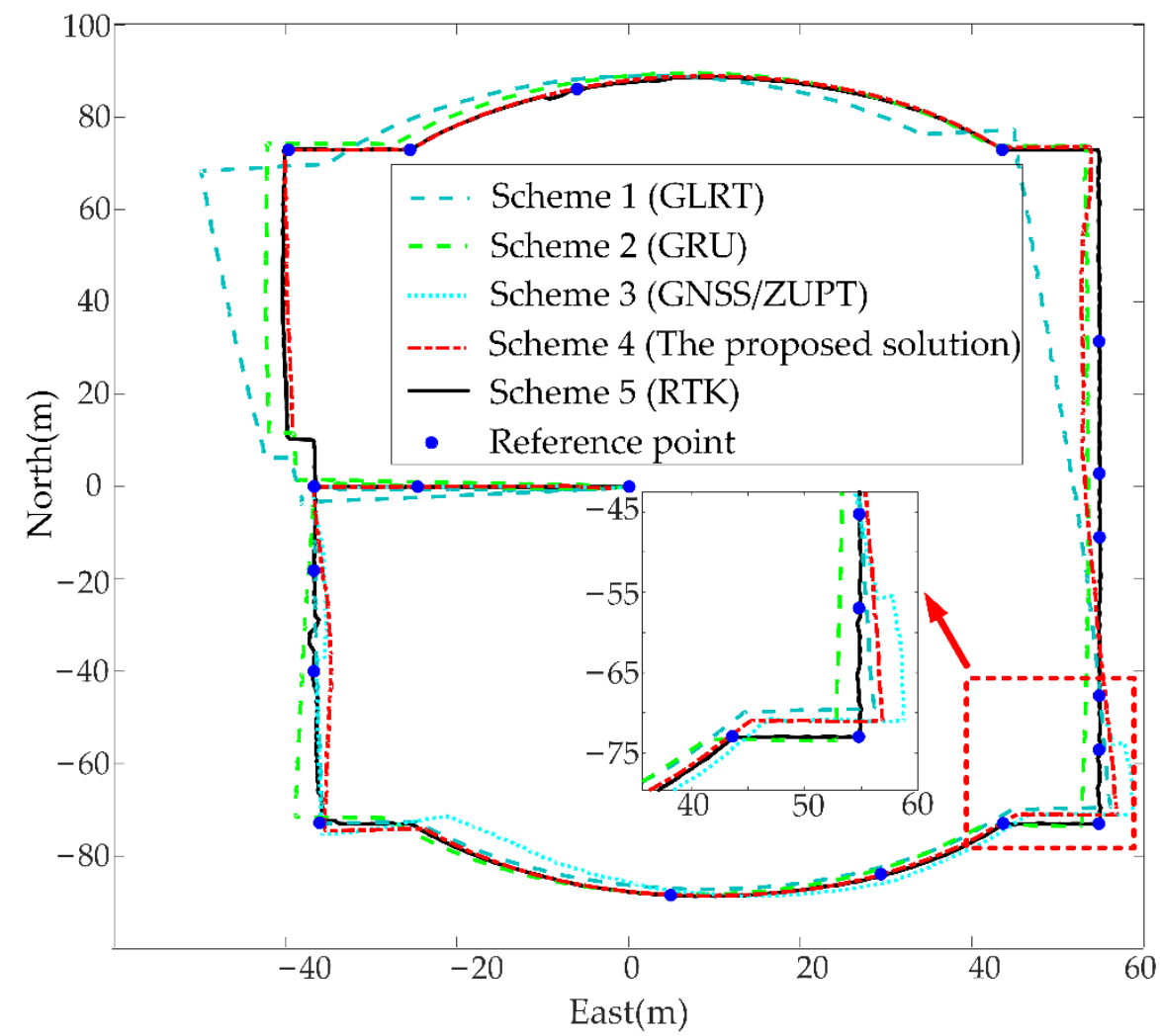

Figure 12. 2D positioning trajectories by different schemes.

As shown in Figure 13, although the position environment is complex, the horizontal error of RTK is lower than $0.5 \mathrm{~m}$ at all of the reference points. Because of the high precision of RTK, the trajectory of RTK is taken as the reference trajectory. In Figure 13, we randomly select 18 points in the test path to test the accuracy of the algorithm. The proposed algorithm obtained lower position error than Schemes 1-3 at most of the reference points, noting that when the INS obtained a large error, the adaptive algorithm may increase the position error of the GNSS/PDR algorithm, such as the position error at reference point 4 . However, in most cases, GNSS has larger position error than INS due to environmental interference; the adaptive algorithm can effectively decrease the position error when GNSS obtained larger error, such as the position error at reference points 6, 7, and 8. The position error of Schemes 1-4 is shown in Table 4 . It can be seen from Table 4 that the RMSE of the four schemes are $6.08 \mathrm{~m}, 3.21 \mathrm{~m}, 1.79 \mathrm{~m}$, and $1.37 \mathrm{~m}$ respectively and the maximum errors of the four schemes are $11.48 \mathrm{~m}, 6.02 \mathrm{~m}, 4.98 \mathrm{~m}$, and $2.79 \mathrm{~m}$ respectively. Compared with the other three schemes, RMSE of the proposed scheme are decreased by $77 \%, 57 \%$, and $23 \%$ respectively and the maximum errors of the proposed scheme are decreased by $75 \%, 53 \%$, and $43 \%$ respectively. CDF of the horizontal error are shown Figure 14. It can be seen from Figure 14 that $99 \%$ of the horizontal error of the proposed scheme is smaller than $2.71 \mathrm{~m}$. For several algorithms used to compare with the proposed scheme, some algorithms do not integrate GNSS position information, so the result obtained will be better than the proposed algorithm, but due to the characteristics of inertial navigation, the accuracy of these algorithms will diverge over time. Generally speaking, the accuracy of the proposed algorithm is better than other algorithms. 


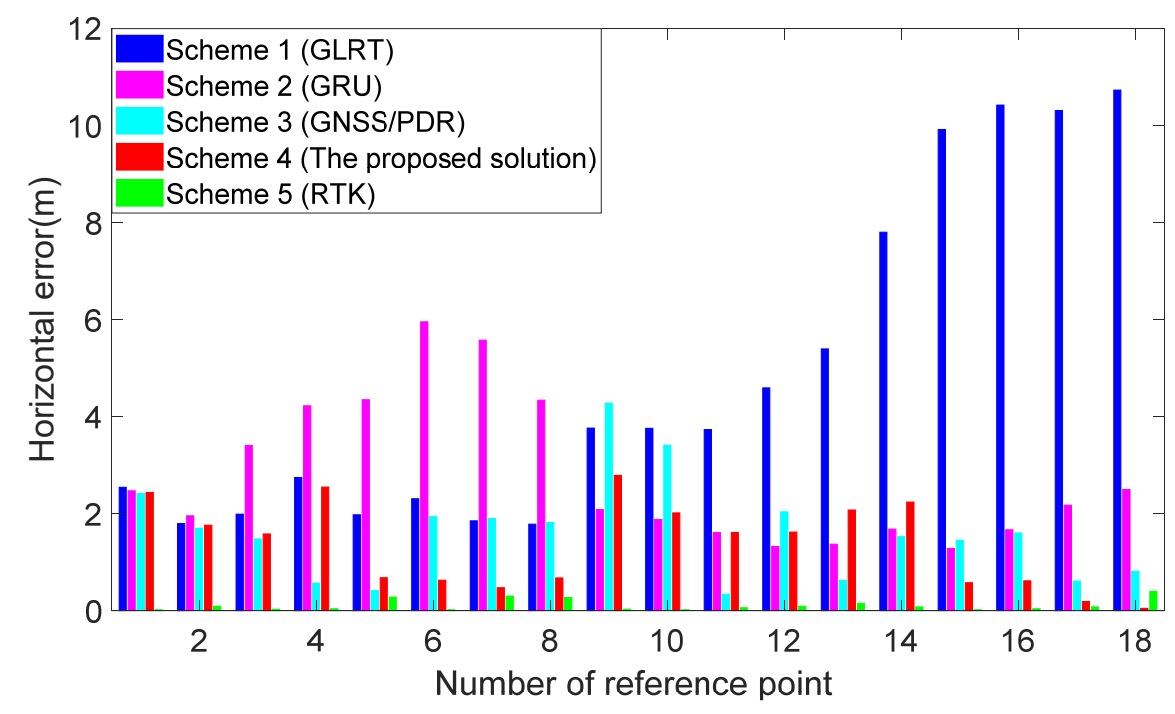

Figure 13. Horizontal error statistics of different schemes.

Table 4. Horizontal position error (m) obtained by different schemes.

\begin{tabular}{ccc}
\hline & Maximum Error & RMSE \\
\hline Scheme 1 & 11.48 & 6.08 \\
Scheme 2 & 6.02 & 3.21 \\
Scheme 3 & 4.98 & 1.79 \\
Scheme 4 & 2.79 & 1.37 \\
\hline
\end{tabular}

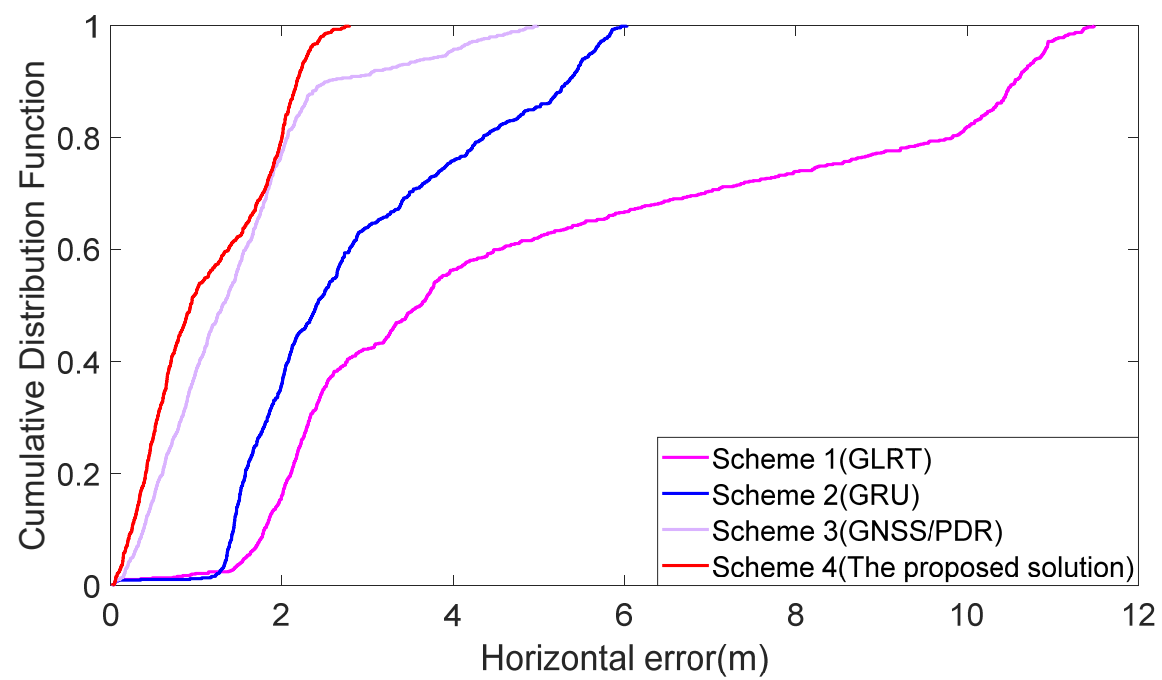

Figure 14. CDF of horizontal error obtained by different schemes.

\subsection{Indoor Elevation Performance Verification of the Proposed Algorithm}

An indoor staircase environment is carried out to verify the elevation performance of the proposed algorithm. The proposed algorithm will not execute GNSS measurement update as there is no GNSS signal in the indoor environment. The experimental site is shown in Figure 15 in this experiment; the experimenter went upstairs from the fifth floor to the twelfth floor, then went downstairs to the fifth floor. The height difference of each floor has been determined in advance by multiple measuring and only counts the elevation error when the experimenter falls on each floor of the staircase. Three schemes are compared. Scheme 1: use ZUPT to judge elevation; Scheme 2: use the barometer to judge elevation; Scheme 3: ZUPT/ barometer fusion algorithm. The test result is shown in Figure 16. 


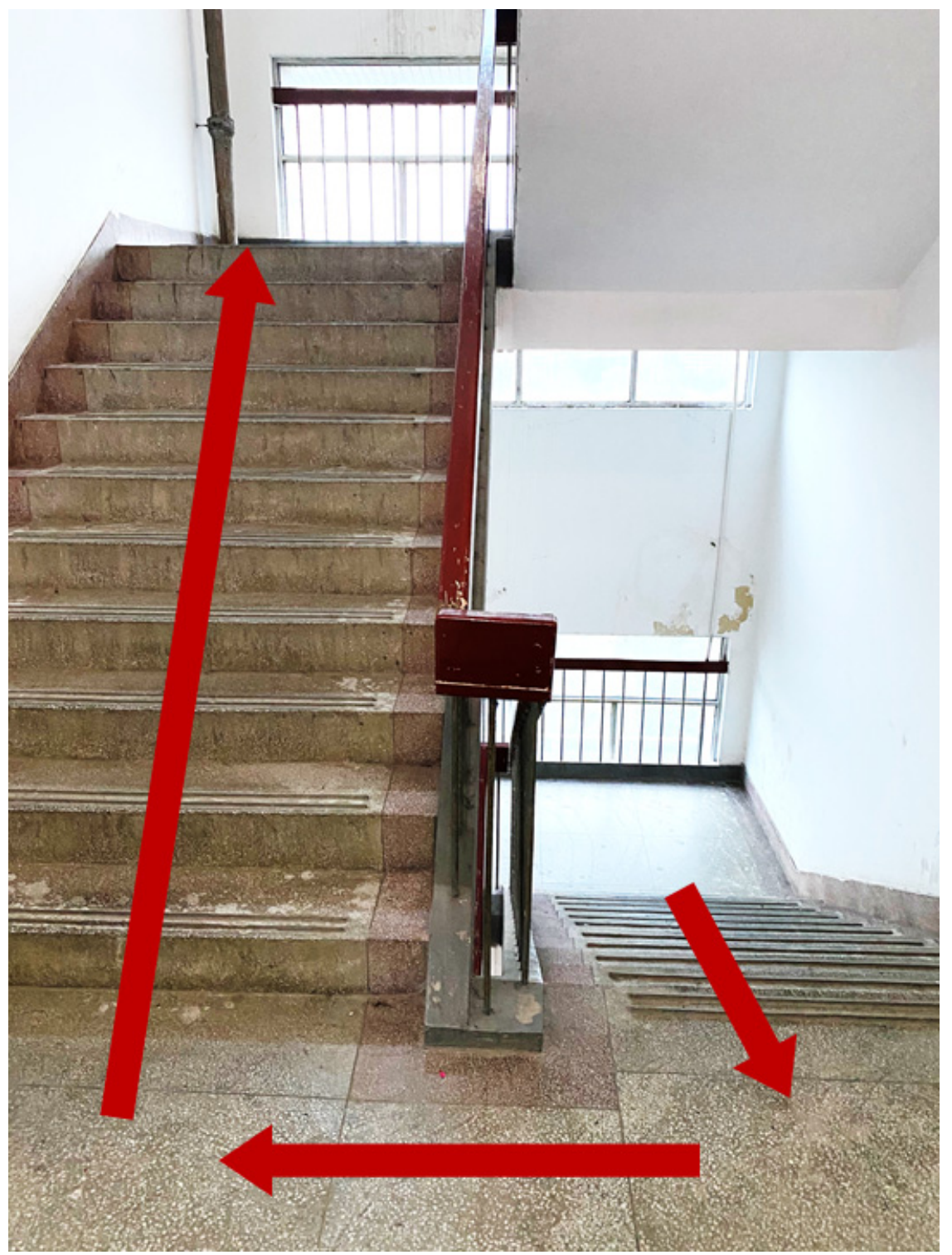

Figure 15. Experimental site e of indoor experiment.

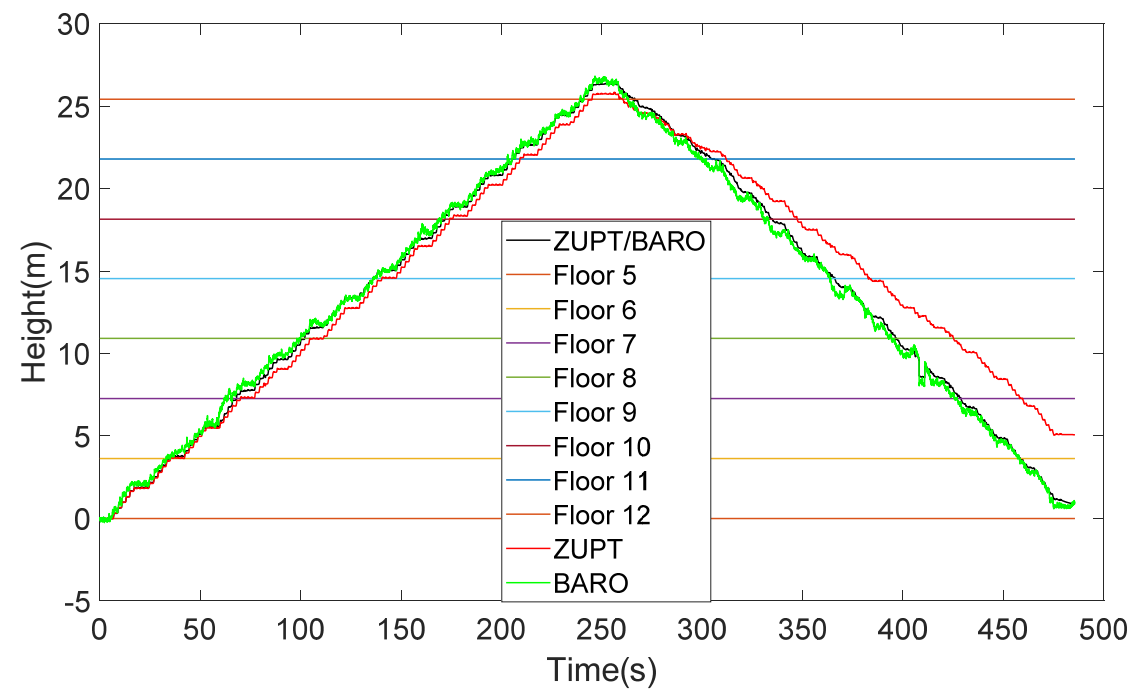

Figure 16. Height comparison of different methods. 
It can be seen from Figure 16 that the barometer can basically reflect the elevation, but the robustness is not enough, and there are many burrs. ZUPT has no large burrs, but the error diverges with time. The result of the adaptive combination of the PDR and barometer can reflect the elevation well without large error. As shown in Table 5, RMSE of these three schemes are $1.62 \mathrm{~m}, 1.25 \mathrm{~m}$, and $0.96 \mathrm{~m}$, respectively. The maximum errors of these schemes are $3.3 \mathrm{~m}, 1.95 \mathrm{~m}$, and $1.28 \mathrm{~m}$, respectively. Compared with other two schemes, RMSE of the proposed scheme are decreased by $41 \%$ and $24 \%$, respectively, and the maximum errors of the proposed scheme are decreased by $61 \%$ and $34 \%$, respectively.

Table 5. Elevation error (m) obtained by different schemes.

\begin{tabular}{ccc}
\hline & MAX & RMSE \\
\hline Scheme 1 & 3.3 & 1.62 \\
Scheme 2 & 1.95 & 1.25 \\
Scheme 3 & 1.28 & 0.96 \\
\hline
\end{tabular}

\section{Conclusions}

A single-frequency single-antenna GNSS/PDR robust integration scheme is proposed, which provides continuous and accurate pedestrian navigation by fusing GNSS and MEMS sensors. Key technologies of the proposed scheme are discussed, including improved carrier phase smoothing pseudo-range GNSS single-point positioning, GRU-based zerovelocity detection, and adaptive fusion algorithm of GNSS and PDR. Kinematic experiments in outdoor open environments and complex environments show that the proposed scheme can combine the advantages of GNSS and INS, achieving pedestrian navigation position error lower than $1.5 \mathrm{~m}$. The proposed GRU-based zero-velocity detection model can only detect ordinary motion patterns. However, due to the limitation of the data set, this model cannot detect the zero-velocity state in complex motion mode. In the future, we will study the zero-velocity detection model under complex motion mode. In summary, this integrated scheme promotes the realization of high-precision positioning of public pedestrians in an urban environment, and it provides technical reference for accurate and continuous acquisition of public pedestrian location information.

Author Contributions: Conceptualization, J.J. and D.X.; methodology, J.J., D.X. and J.W.; software, D.X.; validation, J.J. and D.X.; formal analysis, J.J. and D.X.; investigation, J.J. and D.X.; resources, J.J. and D.X.; writing —original draft preparation, J.J. and D.X.; writing-review and editing, J.J., D.X., P.Y., Y.T., J.W., C.Z. and J.L.; visualization, J.W.; supervision, J.W. and D.X.; project administration, P.Y.; funding acquisition, J.J. All authors have read and agreed to the published version of the manuscript.

Funding: This research was funded by the National Key Research and Development Program of China (2018YFB0505200 and 2018YFB0505201), the Fundamental Research Funds for the Central Universities (2042018kf0253).

Institutional Review Board Statement: Not applicable.

Informed Consent Statement: Not applicable.

Data Availability Statement: Not applicable.

Acknowledgments: Thank you very much for the equipment support of Jinguang Jiang's research group, for his driving experiment for this research, and for the technical support of Fangning Zhang, Peihui Xie, Yanan Tang, Jiaji Wu, and Chao Zhang.

Conflicts of Interest: The authors declare no conflict of interest. 


\section{References}

1. Han, H.; Wang, J. Robust GPS/BDS/INS tightly coupled integration with atmospheric constraints for long-range kinematic positioning. GPS Solut. 2017, 21, 1285-1299. [CrossRef]

2. Kim, Y.; Hwang, Y.; Choi, S.; Lee, J. Height estimation scheme of low-cost pedestrian dead-reckoning system using Kalman Filter and walk condition estimation algorithm. In Proceedings of the 2013 IEEE/ASME International Conference on Advanced Intelligent Mechatronics, Wollongong, NSW, Australia, 9-12 July 2013; pp. 1492-1497.

3. Chiang, K.-W.; Chang, H.-W.; Li, Y.-H.; Tsai, G.-J.; Tseng, C.-L.; Tien, Y.-C.; Hsu, P.-C. Assessment for INS/GNSS/Odometer/Barometer Integration in Loosely-Coupled and Tightly-Coupled Scheme in a GNSS-Degraded Environment. IEEE Sens. J. 2020, 20, 3057-3069. [CrossRef]

4. Yan, P.H.; Jiang, J.G.; Zhang, F.N.; Xie, D.P.; Wu, J.J.; Zhang, C.; Tang, Y.A.; Liu, J.N. An Improved Adaptive Kalman Filter for a Single Frequency GNSS/MEMS-IMU/Odometer Integrated Navigation Module. Remote Sens. 2021, 13, 4317. [CrossRef]

5. Yan, P.H.; Jiang, J.G.; Tang, Y.A.; Zhang, F.N.; Xie, D.P.; Wu, J.J.; Liu, J.H.; Liu, J.N. Dynamic Adaptive Low Power Adjustment Scheme for Single-Frequency GNSS/MEMS-IMU/Odometer Integrated Navigation in the Complex Urban Environment. Remote Sens. 2021, 13, 3236. [CrossRef]

6. Pulido Herrera, E.; Kaufmann, H.; Secue, J.; Quirós, R.; Fabregat, G. Improving data fusion in personal positioning systems for outdoor environments. Inf. Fusion 2013, 14, 45-56. [CrossRef]

7. Noureldin, A.; El-Shafie, A.; Bayoumi, M. GPS/INS integration utilizing dynamic neural networks for vehicular navigation. Inf. Fusion 2011, 12, 48-57. [CrossRef]

8. Cao, H.J.; Wang, Y.J.; Bi, J.X. Smartphones: 3D Indoor Localization Using Wi-Fi RTT. IEEE Commun. Lett. 2021, 25, 1201-1205. [CrossRef]

9. Tian, Q.; Wang, K.I.K.; Salcic, Z. A Low-Cost INS and UWB Fusion Pedestrian Tracking System. IEEE Sens. J. 2019, 19, 3733-3740. [CrossRef]

10. Seco, F.; Jiménez, A.R. Smartphone-based cooperative indoor localization with RFID technology. Sensors 2018, 18, 266. [CrossRef]

11. Santos, D.; Gonçalves, P. Attitude Determination of Multirotor Aerial Vehicles Using Camera Vector Measurements. J. Intell. Robot. Syst. 2016, 86, 1-11. [CrossRef]

12. Ward, N. Development and testing of a radar target enhancer for navigation buoys. J. Navig. 1995, 48, 70-76. [CrossRef]

13. Zhu, R.W.; Wang, Y.; Cao, H.; Yu, B.; Gan, X.; Huang, L.; Zhang, H.; Li, S.; Jia, H.; Chen, J. RTK/Pseudolite/LAHDE/IMU-PDR Integrated Pedestrian Navigation System for Urban and Indoor Environments. Sensor 2020, 20, 1791. [CrossRef]

14. Xin, L.; Jian, W.; Liu, C. A Bluetooth/PDR Integration Algorithm for an Indoor Positioning System. Sensors 2015, 15, 24862-24885.

15. Cavallo, F.; Sabatini, A.M.; Genovese, V. A step toward GPS/INS personal navigation systems: Real-time assessment of gait by foot inertial sensing. In Proceedings of the 2005 IEEE/RSJ International Conference on Intelligent Robots and Systems, Edmonton, AB, Canada, 2-6 August 2005; pp. 1187-1191.

16. Polak, L.; Rozum, S.; Slanina, M.; Bravenec, T.; Fryza, T.; Pikrakis, A. Received Signal Strength Fingerprinting-Based Indoor Location Estimation Employing Machine Learning. Sensors 2021, 21, 4605. [CrossRef]

17. Sun, B.; Fan, Q.; Wu, Y.; Sun, Y. Foot-mounted pedestrian navigation technology based on tightly coupled PDR/UWB. Transducer Microsyst. Technol. 2017, 36, 43-47, 50.

18. Brigante, C.; Abbate, N.; Basile, A.; Faulisi, A.C.; Sessa, S. Towards Miniaturization of a MEMS-Based Wearable Motion Capture System. IEEE Trans. Ind. Electron. 2011, 58, 3234-3241. [CrossRef]

19. Qiu, S.; Wang, Z.; Zhao, H.; Qin, K.; Li, Z.; Hu, H. Inertial/magnetic sensors based pedestrian dead reckoning by means of multi-sensor fusion. Inf. Fusion 2017, 39, 108-119. [CrossRef]

20. Shi, L.-F.; Zhao, Y.-L.; Liu, G.-X.; Chen, S.; Wang, Y.; Shi, Y.-F. A Robust Pedestrian Dead Reckoning System Using Low-Cost Magnetic and Inertial Sensors. IEEE Trans. Instrum. Meas. 2019, 68, 2996-3003. [CrossRef]

21. $\mathrm{Wu}, \mathrm{Z} . ; \mathrm{Hu}, \mathrm{X}$.; $\mathrm{Wu}, \mathrm{M}$.; Cao, J. Constrained total least-squares calibration of three-axis magnetometer for vehicular applications. Meas. Sci. Technol. 2013, 24. [CrossRef]

22. Springmann, J.C.; Cutler, J.W. Attitude-Independent Magnetometer Calibration with Time-Varying Bias. J. Guid. Control Dyn. 2011, 35, 1080-1088. [CrossRef]

23. Deng, Z.; Sun, M.; Wang, B.; Fu, M. Analysis and Calibration of the Nonorthogonal Angle in Dual-Axis Rotational INS. IEEE Trans. Ind. Electron. 2017, 64, 4762-4771. [CrossRef]

24. Wang, Y.; Shkel, A.M. Adaptive Threshold for Zero-Velocity Detector in ZUPT-Aided Pedestrian Inertial Navigation. IEEE Sens. Lett. 2019, 3. [CrossRef]

25. Foxlin, E. Pedestrian Tracking with Shoe-Mounted Inertial Sensors. IEEE Comput. Graph. Appl. 2005, 25, 38-46. [CrossRef] [PubMed]

26. Woyano, F.; Lee, S.; Park, S. Evaluation and comparison of performance analysis of indoor inertial navigation system based on foot mounted IMU. In Proceedings of the 2016 18th International Conference on Advanced Communication Technology (ICACT), Phoenix Park, PyeongChang, Korea, 31 January-3 February 2016; pp. 792-798.

27. Skog, I.H.; Nilsson, P.; Rantakokko, J.-O. Zero-Velocity Detection-An Algorithm Evaluation. IEEE Trans. Bio-Med. Eng. 2010, 57, 2657-2666. [CrossRef]

28. Wang, Z.; Zhao, H.; Qiu, S.; Gao, Q. Stance-Phase Detection for ZUPT-Aided Foot-Mounted Pedestrian Navigation System. IEEE/ASME Trans. Mechatron. 2015, 20, 3170-3181. [CrossRef] 
29. Liu, H.f.; Ren, W.; Zhang, T.; Gong, J.; Liang, J.M.; Liu, B.; Shi, J.W.; Huang, Z. An adaptive selection algorithm of threshold value in zero velocity updating for personal navigation system. In Proceedings of the 33rd Chinese Control Conference, Nanjing, China, 28-30 July 2014; pp. 1035-1038.

30. Wang, Q.; Guo, Z.; Sun, Z.; Cui, X.; Liu, K. Research on the Forward and Reverse Calculation Based on the Adaptive Zero-Velocity Interval Adjustment for the Foot-Mounted Inertial Pedestrian-Positioning System. Sensors 2018, 18, 1642. [CrossRef]

31. Callmer, J.; Törnqvist, D.; Gustafsson, F. Probabilistic stand still detection using foot mounted IMU. In Proceedings of the 2010 13th International Conference on Information Fusion, Edinburgh, UK, $26-29$ July 2010; pp. 1-7.

32. Wagstaff, B.; Peretroukhin, V.; Kelly, J. Improving foot-mounted inertial navigation through real-time motion classification. In Proceedings of the 2017 International Conference on Indoor Positioning and Indoor Navigation (IPIN), Sapporo, Japan, 18-21 September 2017; pp. 1-8.

33. Wagstaff, B.; Kelly, J. LSTM-Based Zero-Velocity Detection for Robust Inertial Navigation. In Proceedings of the 2018 International Conference on Indoor Positioning and Indoor Navigation (IPIN), Nantes, France, 24-27 September 2018; pp. 1-8.

34. Guo, R.; Hu, X.; Chang, Z.; Cao, Y.; Wang, A.; Wu, X.; Dong, E. Comparison between CNMC and hatch filter \& its precision analysis for BDS precise relative positioning. Sci. Sin. Phys. Mech. Astron. 2015, 45, 079508. [CrossRef]

35. Chebir, S.; Aidel, S.; Rouabah, K.; Attia, S.; Flissi, M. GNSS Signals Acquisition and Tracking in Unfavorable Environment. Radioengineering 2018, 27, 557-571. [CrossRef]

36. Park, B.; Sohn, K.; Kee, C. Optimal Hatch Filter with an Adaptive Smoothing Window Width. J. Navig. 2008, 61, 435-454. [CrossRef]

37. Fernando, T.; Denman, S.; Sridharan, S.; Fookes, C. Soft plus Hardwired attention: An LSTM framework for human trajectory prediction and abnormal event detection. Neural Netw. 2018, 108, 466-478. [CrossRef]

38. Zheng, Z.; Chen, W.; Wu, X.; Chen, P.; Liu, J. LSTM network: A deep learning approach for short-term traffic forecast. IET Intell. Transp. Syst. 2017, 11, 68-75.

39. Kingma, D.P.; Ba, J. Adam: A method for stochastic optimization. arXiv 2014, arXiv:1412.6980. 